\title{
Parabolic tendons in prestressed concrete - how accurate are equivalent loads?
}

\author{
Matti Pajari
}

Summary The mechanical effects of a parabolic tendon can be modeled by replacing the tendon by external loads applied to the concrete. The intensity of these loads depends on the prestressing force $P$ and curvature of the tendons. These two factors are also interrelated because the losses of prestress vary with the curvature. The structural analysis can be simplified by approximating that, between the anchors, the line load generated by the tendon against the concrete is constant, perpendicular to the centroidal axis of the beam and equal to $P / R$ where $R$ is the radius of curvature of the parabola at its vertex. This approximation is one of the key issues in the textbooks but normally not properly justified. In this paper, the mathematical background for the approximation is formulated. Some typical tendon layouts are analyzed to evaluate the approximation error. The error proved to be insignificant for simple beams. For cantilever and continuous beams more accurate methods in the final design are recommended.

Key words: parabolic tendon, equivalent load, post-tensioning, prestress, concrete, structure

Received: 20 December 2020. Accepted: 14 June 2021. Published online: 15 November 2021.

\section{Introduction}

Parabolic prestressing tendons embedded in concrete members are mainly used to resist uniformly or almost uniformly distributed loads. The mechanical behaviour of such tendons can be easily illustrated by the concept of equivalent loads. The concrete component is regarded as a free body, and the effects of each tendon are replaced by equivalent loads, i.e. by external loads applied to the concrete: point loads at the anchors and a line load between them. The textbooks, e.g., Gilbert \& Mickleborough [1], p. 9, and Lin \& Burns [2], p. 21, generally state that this line load is equivalent to a constant, external line load perpendicular to the centroidal axis of the beam and equal to $q=2 \mathrm{~Pa}$ where $P$ is the tendon force and $a$ denotes the coefficient of the second-degree term of the parabola. In the rest of this paper, the counterforce of $q$ exerted on the concrete is called approximate or traditional equivalent load or simply equivalent load. Even though it is not explicitly stated, the textbooks give an impression that if a concrete beam is shallow enough to justify the use of elementary beam theory, the traditional equivalent load is a safe approximation despite the fact that, due to the losses of prestress, $P$ varies along the length of the tendon. The proper value of $P$ to be used for in the equivalent load is subject for engineering judgement. Since the key point of the present study is to illustrate the error when the varying curvature of a parabolic tendon is modeled by its maximum value, and idealized tendon with constant $P$ is used in the considerations. 
A great number of peer reviewed articles about formulation and application of loads equivalent to the parabolic and other loads have been published. It is generally assumed that the eccentricity of the tendon is much smaller than the span length(s) of the beam. In some cases, the consequences of this and other simplifying assumptions on the mechanical equilibrium have qualitatively been mentioned, but to the author's knowledge, no quantitative comparison about the accuracy of the equivalent load approach has been published.

\section{Actions due to curved tendon}

Actions exerted to the concrete by a curved tendon are equal but opposite to the actions which the concrete exerts to the tendon. The latter actions are considered in this section.

Assume that $u=u(x)$ gives the vertical position of an in-plane tendon subjected to an axial force $P$ at the ends. $s$ denotes the coordinate measured along the tendon. In the absence of bond and friction, there are no tangential external forces affecting the tendon between the anchors. Let $q=q(s)$ be the perpendicular force per unit length of the tendon and $\alpha=\alpha(x)$ the angle between the horizontal axis and the tendon, see Figure 1. In Figure 2, the free body diagram for a short cut of the tendon with length $\Delta s$ is shown.

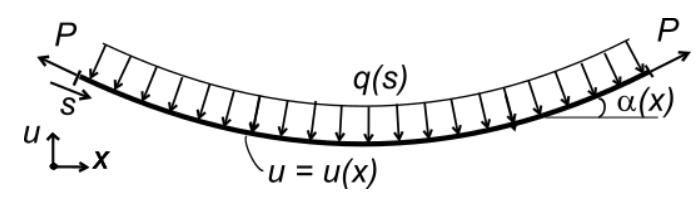

Figure 1. Forces affecting the idealized tendon.

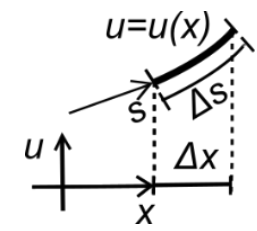

a)

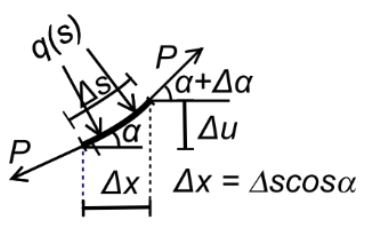

(b)

Figure 2. a) Notation. b) Forces on a short cut of tendon.

For a small increment $\Delta s$, we obtain

$$
\begin{gathered}
\Delta s \approx \frac{\Delta x}{\cos \alpha} \\
\frac{d u}{d x}=\tan \alpha \\
\frac{d^{2} u}{d x^{2}}=\frac{d(\tan \alpha)}{d \alpha} \frac{d \alpha}{d x}=\frac{1}{\cos ^{2} \alpha} \frac{d \alpha}{d x} \\
\Delta \alpha \approx \frac{d \alpha}{d x} \Delta x=\frac{d^{2} u}{d x^{2}} \cos ^{3} \alpha \Delta s
\end{gathered}
$$

The resultant of the horizontal component of $q(s)$ in Figure 2 equals to

$$
Q_{h o r} \approx q(s) \sin (\alpha) \Delta s
$$

The equilibrium of horizontal forces gives 


$$
\begin{gathered}
P \cos \alpha \approx q(s) \sin (\alpha) \Delta s+P \cos (\alpha+\Delta \alpha) \\
q(s) \sin (\alpha) \Delta s \approx-P[\cos (\alpha+\Delta \alpha)-\cos \alpha]
\end{gathered}
$$

Dividing and multiplying the right-hand side by $\Delta \alpha$ and using Equation 4 we obtain

$$
q(s) \sin (\alpha) \Delta s \approx-P \frac{[\cos (\alpha+\Delta \alpha)-\cos \alpha]}{\Delta \alpha} \frac{d^{2} u}{d x^{2}} \cos ^{3} \alpha \Delta s
$$

Divide both sides by $\Delta s$ and let $\Delta \alpha \rightarrow 0$. It follows that

$$
q(s) \sin (\alpha)=-P(-\sin \alpha) \frac{d^{2} u}{d x^{2}} \cos ^{3} \alpha
$$

or, in case $\sin (\alpha) \neq 0$

$$
q(s)=P \frac{d^{2} u}{d x^{2}} \cos ^{3} \alpha
$$

When $\sin (\alpha)=0$, the same result is obtained by considering the equilibrium of vertical forces. The vertical and horizontal components of $q$ are

$$
\begin{aligned}
& q_{v}(s)=q(s) \cos \alpha=P \frac{d^{2} u}{d x^{2}} \cos ^{4} \alpha \\
& q_{h}(s)=q(s) \sin \alpha=P \frac{d^{2} u}{d x^{2}} \cos ^{3} \alpha \sin \alpha
\end{aligned}
$$

In numeric calculations it is more convenient to use the vertical and horizontal forces per unit length in the $x$-direction:

$$
\begin{aligned}
& q_{v, x}=P \frac{d^{2} u}{d x^{2}} \cos ^{4} \alpha \frac{\Delta s}{\Delta x}=P \frac{d^{2} u}{d x^{2}} \cos ^{3} \alpha \\
& q_{h, x}=P \frac{d^{2} u}{d x^{2}} \cos ^{3} \alpha \sin \alpha \frac{\Delta s}{\Delta x}=P \frac{d^{2} u}{d x^{2}} \cos ^{2} \alpha \sin \alpha
\end{aligned}
$$

\section{Applications}

\section{Circular Tendon}

To check the theory in the simplest possible case, it is first applied to a circular arch for which the exact solution is well-known.

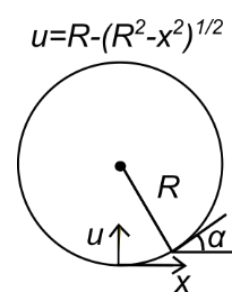

Figure 3. Notation. 
For a circular arch, see Figure 3 for the symbols, the well-known exact solution $q=P / R$ is also obtained from Equation 15:

$$
q(s)=P \frac{d^{2} u}{d x^{2}} \cos ^{3} \alpha=P\left(\frac{1}{\sqrt{R^{2}-x^{2}}}+\frac{x^{2}}{\left(\sqrt{R^{2}-x^{2}}\right)^{3}}\right)\left(\frac{\left(\sqrt{R^{2}-x^{2}}\right)^{3}}{R^{3}}\right)=\frac{P}{R}
$$

\section{Parabolic Tendon}

For a parabola $u=a x^{2}+b x+c$,

$$
q(s)=P \frac{d^{2} u}{d x^{2}} \cos ^{3} \alpha=2 P a \cos ^{3} \alpha
$$

The vertical and horizontal forces per unit length in the $x$-direction are

$$
\begin{gathered}
q_{v, x}=2 \operatorname{Pacos}^{3} \alpha \\
q_{h, x}=2 \operatorname{Pacos}^{2} \alpha \sin \alpha
\end{gathered}
$$

When $\alpha \approx 0, \cos \alpha \approx 1$ and $\sin \alpha \approx 0$. It follows that $\tan \alpha=u^{\prime} \approx 0$. In such a case

$$
\begin{aligned}
& q_{v, x} \approx 2 P a \\
& q_{h, x} \approx 0
\end{aligned}
$$

Figure 4 illustrates the parameters of parabola

$$
u(x)=\frac{4 h}{L^{2}}\left(x-\frac{L}{2}\right)^{2}
$$

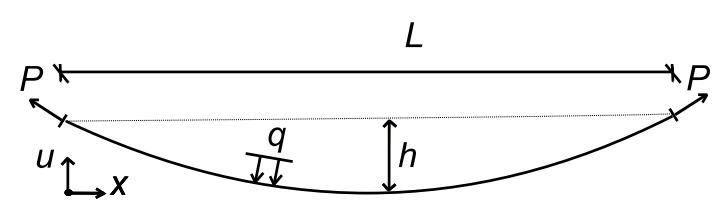

Figure 4. Notation for a parabolic tendon.

For this parabola

$$
\begin{gathered}
q_{v, x}=P \frac{d^{2} u}{d x^{2}} \cos ^{3} \alpha=\frac{8 P h}{L^{2}} \cos ^{3} \alpha \approx \frac{8 P h}{L^{2}} \\
q_{h, x}=P \frac{d^{2} u}{d x^{2}} \cos ^{2} \alpha \sin \alpha=\frac{8 P h}{L^{2}} \cos ^{2} \alpha \sin \alpha \approx 0
\end{gathered}
$$

In the outermost spans of a continuous beam, it is advantageous to use tendon profiles which are not symmetric with respect to the midpoint of span. This can be done, e.g., by adding a linear term $k x+b$ to the expression of $u(x)$ which then becomes

$$
u(x)=\frac{4 h}{L^{2}}\left(x-\frac{L}{2}\right)^{2}+k x+b
$$


In daily practice it is assumed that $q$ for tendon profiles defined by Equations 21 and 24 is the same. This is justified e.g. in the classical textbook of Lin \& Burns [2], p. 372, by stating that “...by linear transformation, the shape of the line within each span remains unchanged...". Strictly speaking, the radius of curvature for $u=u(x)$ is expressed as

$$
R=\left|\frac{\left(1+u^{2}\right)^{\frac{3}{2}}}{u^{\prime \prime}}\right|
$$

A linear change $k x+b$ with $k \neq 0$ also changes $u$, and the curvature of the tendon. However, when $u$,2 is much smaller than 1 it is justified to write

$$
R \approx\left|\frac{1}{u^{\prime \prime}}\right|=\frac{1}{|2 a|}
$$

Figure 5 illustrates $q_{v, x}$ and $q_{h, x}$ for the parabolic tendon profile shown in Figure 4. In mid-span, the approximate value $2 \mathrm{~Pa}$ equals the correct value of the vertical component $q_{v, x}$ but next to the support the difference is more than $10 \%$. However, a direct comparison of $q_{v, x}$ with $2 \mathrm{~Pa}$ is not relevant because $q_{h, x}$ also affects the bending moment and because the bending moment is not sensitive to transverse loads close to the supports, see Figure 5. The validity of the approximation must be studied by evaluating the bending moment itself, not $q_{v, x}$ alone.

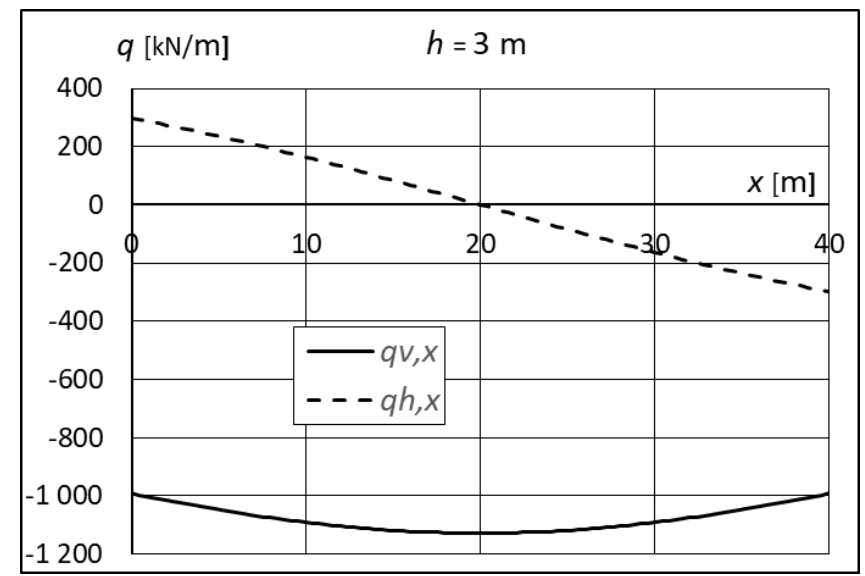

Figure 5. Horizontal $\left(q_{h, x}\right)$ and vertical $\left(q_{v, x}\right)$ component of $q . L=40 \mathrm{~m}$ and $h=3 \mathrm{~m}$.

\section{Numerical comparison of exact and approximate equivalent loads}

\section{Principles of Exact Solution}

Consider first a statically determinate beam shown in Figure 6 in which c.g.c. refers to the centroidal axis of the concrete section.

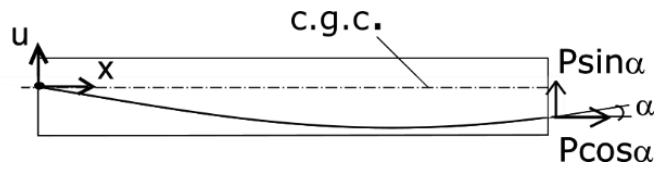

Figure 6. Forces due to $P$ acting on a tendon at an arbitrary section. 
From equilibrium of forces at an arbitrary section, the following action effects on the concrete section are obtained from the free body diagram:

$$
\begin{aligned}
& N(x)=-P \cos \alpha(x) \\
& V(x)=-P \sin \alpha(x) \\
& M(x)=u(x) P \cos \alpha(x)
\end{aligned}
$$

A continuous beam is solved by first removing $n-2$ restrictions where $n$ is the number of supports, then calculating the deflections using Equation (29) for the moment, adding vertical loads which make the total deflections at the removed restrictions equal to zero, and finally superimposing the bending moment due to these vertical loads to the bending moment obtained from Equation (29).

In the following a few beams are compared. Their tendon profiles are smooth, i.e., $u(x)$ is continuously differentiable. For each beam, several eccentricities $h$ of each tendon profile $u$ are considered in such a way that $u(x, h)=h u_{0}(x)$ where $h$ is the depth of the profile. The bending moment $M$ due to the prestressing force $P$ is independent of the concrete cross section and directly proportional to $P$. This implies that the relative error, i.e., the error due to the approximation divided by the reference bending moment $M_{\text {ref }}$ is also independent of $P$. Here $M_{\text {ref }}$ is the maximum absolute value of the exact bending moment $M_{\mathrm{ex}}$ in the zone considered or

$$
M_{\text {ref }}=\max \left\{\left|M_{e x}(x)\right|\right\}
$$

For a simple beam $M_{\text {ref }}$ is obtained at mid-span, for a cantilever beam at the clamped end. In a continuous beam each positive and negative moment zone corresponds to a different $M_{\text {ref. }}$ Since $P$ and the beam cross section do not contribute to the relative error, they are not explicitly given in the following.

\section{Simple Beam}

Consider the hypothetical beam shown in Figure 7. The tendon geometry is given by

$$
u(x)=\frac{4 h}{L^{2}}\left(x-\frac{L}{2}\right)^{2}-h
$$

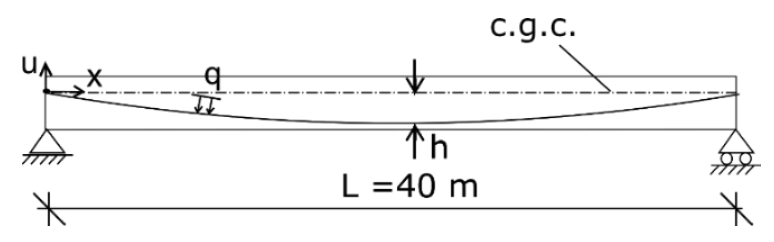

Figure 7. Simple beam with parabolic tendon.

The exact bending moment affecting the concrete at supports and at midspan equals 0 and $-P h$, respectively. Assuming that the vertical uniformly distributed load $q=-2 P a$ we obtain $M(0)=M(L)$ $=0$ and

$$
M(L / 2)=-2 P \frac{4 h}{L^{2}} \frac{L^{2}}{8}=-P h
$$

In other words, the approximation gives the exact bending moment both at supports and at midspan. The approximation overestimates the effect of the vertical component of $q$ but ignores the 
horizontal component. This explains why the bending moment at mid-span can be correctly approximated.

In Figure 8, the exact bending moment calculated by using Equations $(27-29)$ is compared with that due to traditional equivalent load, i.e., transverse constant uniformly distributed load $q=-2 P a=$ $-8 h / L^{2}$. In Figure 8, the error in approximation divided by reference value $M_{\text {ref }}=P h$ is shown for three profile eccentricities $h$.

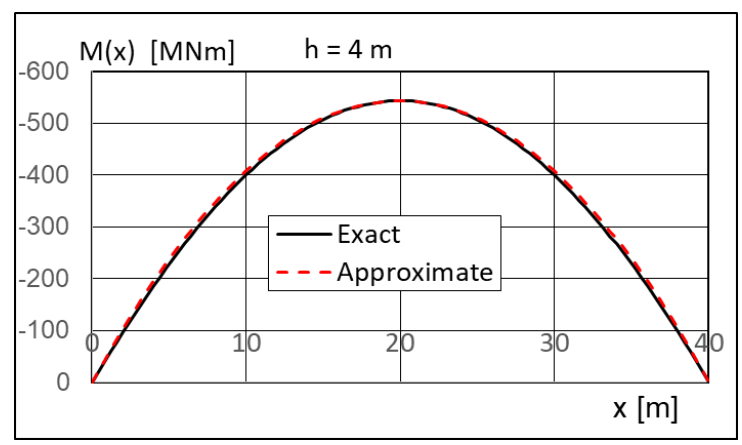

Figure 8. Comparison of approximated and exact bending moment.

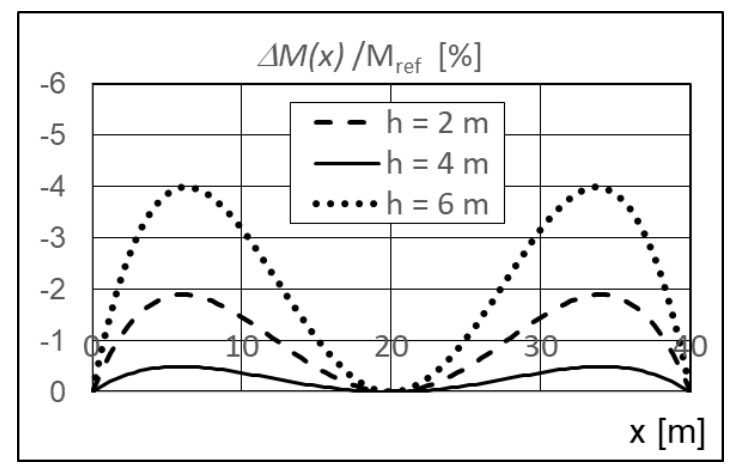

Figure 9. Relative error in approximated bending moment.

The approximation slightly overestimates the effect of prestressing outside the critical zone which is in the mid-zone. This is unimportant as regards the stress analysis. The mid-point camber is also overestimated but only by less than $1 \%$.

The maximum relative error increases with $h$. It occurs close to the supports where the risk of bending failure and cracking in ordinary beam structures is seldom critical. $h$ greater than $6 \mathrm{~m}$ means that application of the elementary beam theory is no more accurate. To conclude, when the beam is shallow enough to enable the use of the elementary beam theory, it is safe to check the bending effects of a parabolic tendon in a simply supported beam using the traditional equivalent loads provided that the losses of prestress are taken into account by a proper manner.

\section{Cantilever Beam}

The beam shown in Figure 10 is prestressed with a tendon for which

$$
u(x)=\frac{h}{L^{2}} x^{2}
$$

The error in the approximated bending moment, see Figs 10 and 11, is more pronounced than in the simply supported beam. This is attributable to the fact that the approximation overestimates the vertical component of $q$ and this effect is not balanced by the asymmetric horizontal forces. Moreover, 
the maximum error not only overestimates the effect of the prestress but also occurs at the critical section.

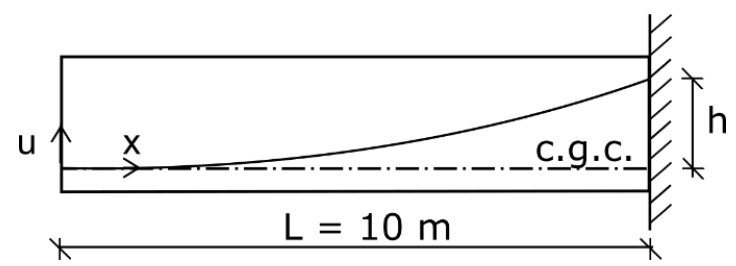

Figure 10. Cantilever beam.

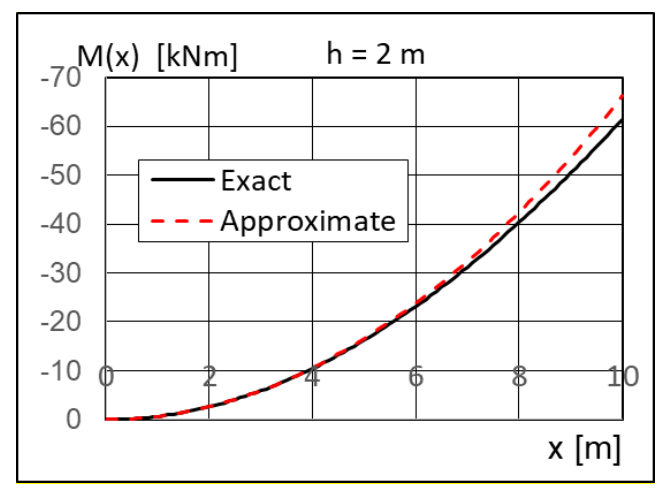

Figure 11. Comparison of exact and approximate bending moment.

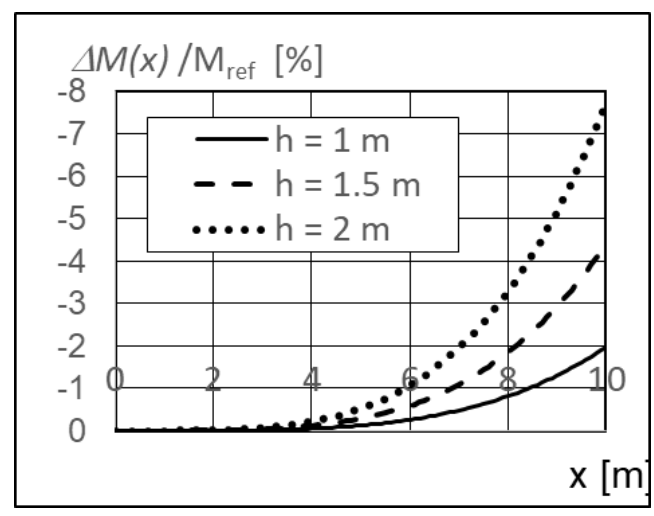

Figure 12. Relative error in cantilever beam.

The camber of the free end of the beam is overestimated by $1.3 \%$ and $5.1 \%$ for $h=1.0 \mathrm{~m}$ and 2.0 $\mathrm{m}$, respectively, when calculated using the elementary beam theory. This is not a very accurate approach for the case $h=2.0 \mathrm{~m}$ but suggests that the relative error in the deflection is smaller than that in the bending moment.

\section{Continuous Beam}

A continuous beam with three spans is shown in Figure 13.

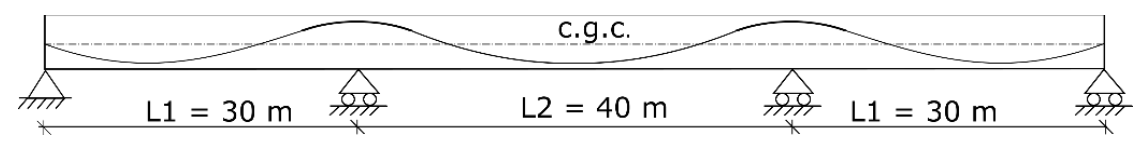

a) 


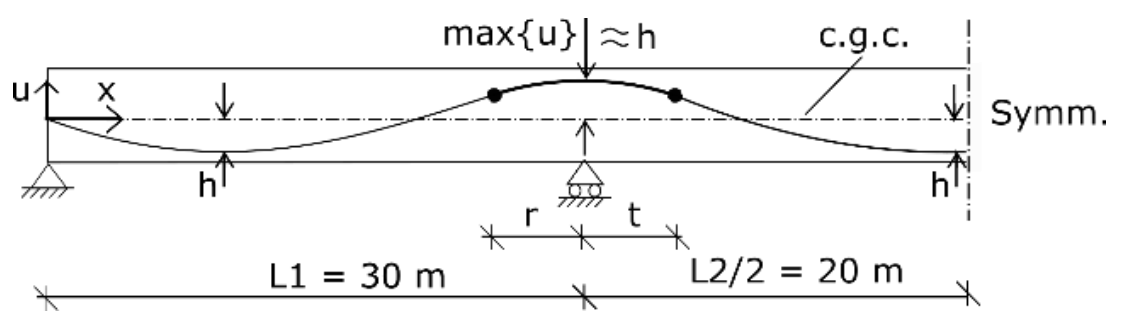

b)

Figure 13. a) Continuous beam. b) Tendon profile.

Using the symbols of Figure 13, the tendon geometry is defined by

$$
\begin{array}{cc}
u(x)=a_{1} x^{2}+b_{1} x & \text { when } x \leq L_{1}-r \\
u(x)=a_{3}\left(x-L_{1}\right)^{2}+b_{3}\left(x-L_{1}\right)+c_{3} & L_{1}-r<x \leq L_{1}+t \\
u(x)=a_{2}\left(x-L_{1}-\frac{L_{2}}{2}\right)^{2}+c_{2} & L_{1}+t<x \leq L_{1}+\frac{L_{2}}{2}
\end{array}
$$

$r+t$ is the length of the concave parabola zone. Cases with $t=4.0 \mathrm{~m}$ and $t=6.5 \mathrm{~m}$ are first considered. Parameters $a_{\mathrm{i}}, b_{\mathrm{i}}, c_{\mathrm{i}}$ and $r$ are chosen in such a way that $u$ is continuously differentiable at $x=L_{1}-r$ and $L_{1}+t$, see Appendix A for the numerical values. The traditional equivalent loads on the concrete, see Figure 14, are

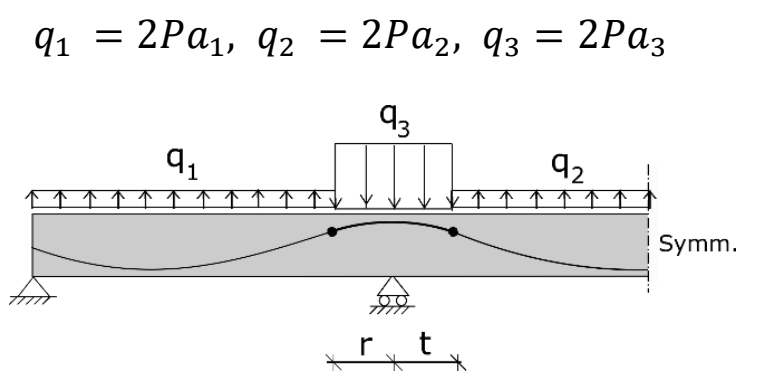

Figure 14. Uniformly distributed equivalent loads.

Figures 15 and 16 compare the bending moments calculated for two lengths of concave tendon profiles keeping the eccentricity of the tendon constant.

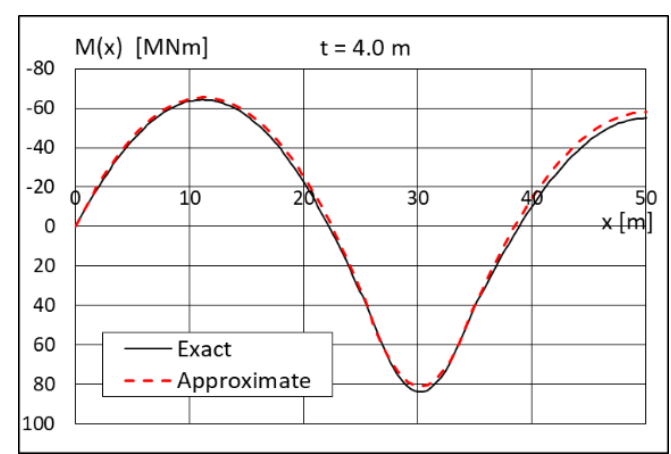

a)

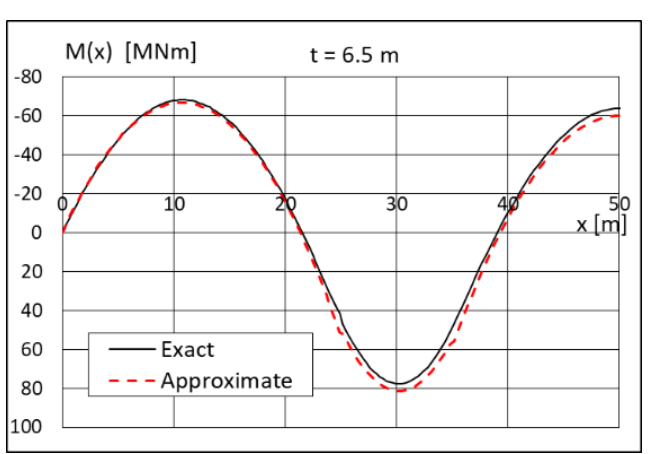

b)

Figure 15. $h=2.0 \mathrm{~m}$. Comparison of exact and approximate bending moment for two lengths of concave tendon zone. 
The moment diagram appears to be sensitive to the length of the concave tendon zone: the approximate moment diagrams for the shorter length $(r+t=3.93+4.0=7.93 \mathrm{~m})$ and longer length $(6.39+6.5=12.39 \mathrm{~m})$ are on the opposite sides of the exact moment diagram. This suggests that there might be an intermediate length which minimizes the error. Indeed, when $r=4.91 \mathrm{~m}$ and $t=$ $5.0 \mathrm{~m}$, the approximate and exact moment diagrams coincide so accurately, that the difference is too small for graphical illustration. The relative error for $h=2.0 \mathrm{~m}$ is illustrated in Figure 16 for $t=4.0$ $\mathrm{m}$ and $5.0 \mathrm{~m}$.

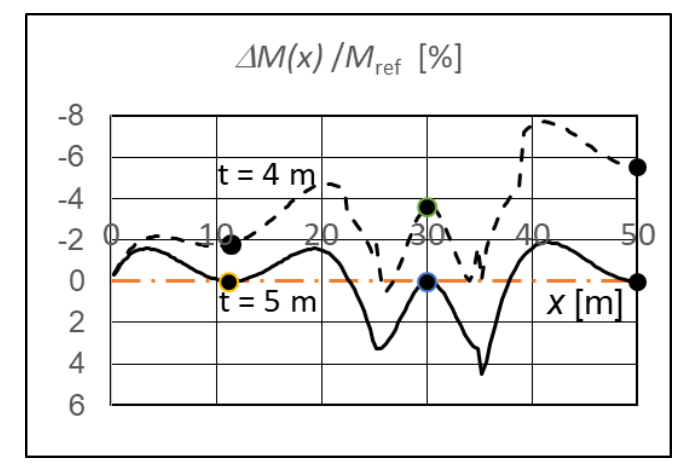

Figure 16. $h=2 \mathrm{~m}$. Relative error in approximated bending moment for two $t$-values.

When a uniformly distributed load is applied to a continuous beam, the negative bending moment at the intermediate supports and the maximum positive bending moment in the span determine the critical zones in the service design. A moderate error in bending moment estimation outside these zones does not have severe consequences. In Figure 16, the location of maxima and minima of the bending moment due to the prestress are indicated by black dots. The critical regions in serviceability design are not far from these dots. With this in mind, the significant approximation error for $t=4.0$ $\mathrm{m}$ is of the order of $4 \%$ and $6 \%$ at the intermediate support and in the middle of the beam, respectively. For $t=6.5 \mathrm{~m}$, see Figure 17, the elative error is less pronounced, and for $t=5.0 \mathrm{~m}$ it is meaningless.

As illustrated in Figure 17, the significant approximation error for the maximum and minimum bending moment is virtually independent of the eccentricity $h$.

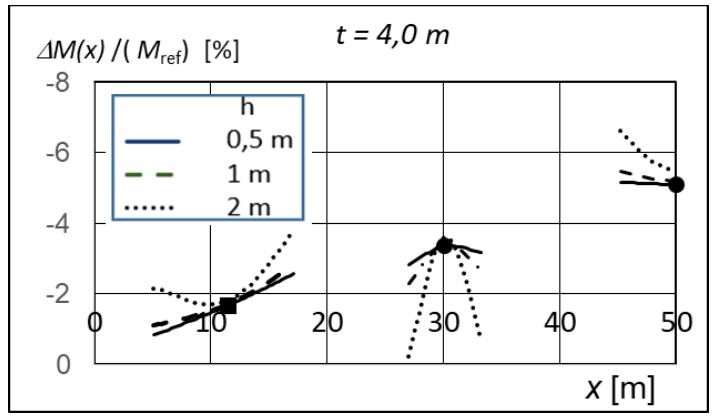

a)

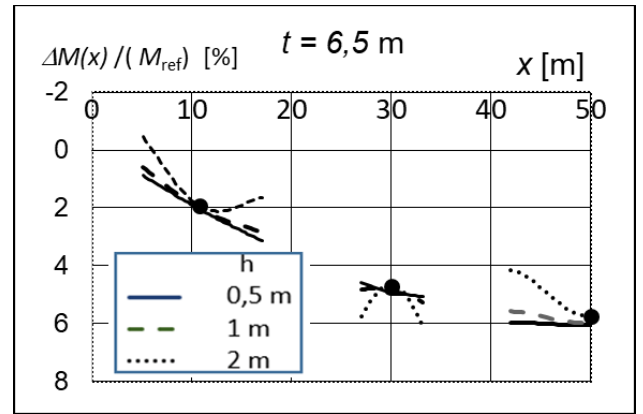

b)

Figure 17. Relative error in approximated bending moment. a) $t=4.0 \mathrm{~m}$. b) $t=6.5 \mathrm{~m}$.

\section{Conclusions}

The curvature of parabola $u(x)=a x^{2}+b x+c$ varies with $x$ and has its maximum at the vertex of the parabola. The traditional concept of equivalent load assumes that between the anchors, the effects of a parabolic tendon on the concrete are equivalent to a constant line load $q=-2 P a$ where $q$ is considered perpendicular to the axis of the beam and $P$ is the prestressing force in the tendon. Since $P$ also varies with $x$ due to the losses of prestress, some properly chosen value of $P$ has to be used to make $q$ constant. 
To evaluate the error in the bending moment due to this approximation, numerical examples have been analyzed assuming idealized tendons with constant axial force along the tendon. Three cases are considered: a simply supported, a cantilever and a continuous beam.

The maximum error in cantilever beams occurs at the fixed end which is the critical cross section in the design. The relative error is proportional to the depth of the tendon profile.

The relative error in simply supported beams is also proportional to the tendon depth, but it vanishes at mid-span and at supports. The maximum deviation from the exact value occurs close to the supports where it is unimportant.

The behaviour of continuous beams with parabolic tendons is more complex than that of a simply supported or cantilever beam. The critical regions in the design are located at intermediate supports and in the span between the supports, but the extreme values of the relative error occur elsewhere. The relative error at the critical sections seems to be almost insensitive to the eccentricity of the tendon profile, but it is sensitive to the length of the concave parabola zone over the intermediate supports.

When elementary beam theory is applied, the depth to span-ratio of the member cannot be too high. Very small radii of curvature are not possible for technical reasons. Due to these two factors, the resulting errors in the analysis based on traditional equivalent loads remain small enough to justify such an approximation in the preliminary design. This may not be the case in the final design of cantilever and continuous beams as shown by a few examples. In any case, more research is needed to find out a reliable answer to the question: When is it safe to apply the traditional concept of equivalent loads to parabolic tendons?

The concept of equivalent loads provide the designers with a useful tool for preliminary design of continuous beams and with an excellent illustration for the actions due to parabolic tendons. Unfortunately, numerous textbooks written so far include no proper justification for the traditional equivalent loads in case of parabolic tendons. The presented "exact" equivalent load distribution is proposed for education to show under which conditions the traditional equivalent loads work properly. It also explains, why a linear change in the tendon geometry of a continuous beam does not change the equivalent loads: the curvature approximated by the second derivative is not affected by the second derivative of the linear change.

\title{
References
}

[1] R. I. Gilbert and N. C. Mickleborough. Design of Prestressed Concrete. Spon Press, New York and London, 2002. ISBN 0-419-16160-0.

[2] T. Y. Lin and N. H. Burns. Design of Prestressed Concrete Structures. John Wiley \& Sons, Inc. New York, 1982. ISBN 0-471-86724-1.

\author{
Matti Pajari \\ Berakon \\ Peräsin 2 B, FI-02320 Espoo, Finland \\ matti.pajari@berakon.fi
}




\section{Appendix A}

\section{Parameters of tendon geometry in continuous beams}

The parameters of the parabolas determining the tendon profile in Figure A1 are solved by setting $u(x)$ and $u^{\prime}(\mathrm{x})$ continuous at $x=L_{1}-r$ and $x=L_{1}+t$.

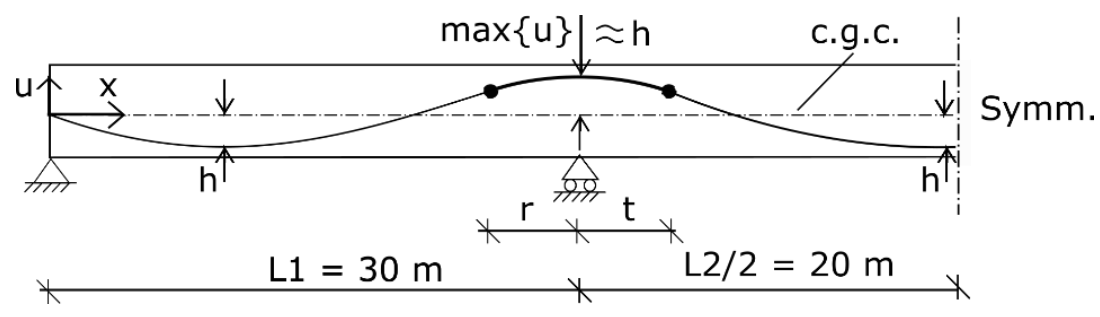

Figure A1. Continuous beam.

$$
\begin{array}{cc}
u(x)=a_{1} x^{2}+b_{1} x & \text { when } \quad \mathrm{x} \leq L_{1}-r \\
u(x)=a_{3}\left(x-L_{1}+r\right)^{2}+b_{3}\left(x-L_{1}+r\right)+c_{3} & L_{1}-r<x \leq L_{1}+t \\
u(x)=a_{2}\left(x-L_{1}-\frac{L_{2}}{2}\right)^{2}+c_{2} & L_{1}+t<x \leq L_{1}+\frac{L_{2}}{2}
\end{array}
$$

Set now $u$ and $u^{\prime}$ continuous at $x=L_{1}-r$ and $\mathrm{x}=L_{1}+t$ :

$$
\begin{gathered}
u\left(L_{1}-r\right)=a_{1}\left(L_{1}-r\right)^{2}+b_{1}\left(L_{1}-r\right)=c_{3} \\
u^{\prime}\left(L_{1}-r\right)=2 a_{1}\left(L_{1}-r\right)+b_{1}=b_{3} \\
u\left(L_{1}+t\right)=a_{3}(r+t)^{2}+b_{3}(r+t)+c_{3}=a_{2}\left(t-\frac{L_{2}}{2}\right)^{2}+c_{2} \\
u^{\prime}\left(L_{1}+t\right)=2 a_{3}(r+t)+b_{3}=2 a_{2}\left(t-\frac{L_{2}}{2}\right)
\end{gathered}
$$

Eliminating $a_{3}$ from Equations (A6) and (A7) gives

$$
(r+t)\left[2 a_{2}\left(t-\frac{L_{2}}{2}\right)+b_{3}\right]-2 a_{2}\left(t-\frac{L_{2}}{2}\right)^{2}-2 c_{2}+2 c_{3}=0
$$

Given $h, t, a_{1}, b_{1}, a_{2}$ and $c_{2}$ and using Equations (A4) and (A5) for $b_{3}$ and $c_{3} . r$ becomes the only unknown in Equation (A8). Solving for $r$ also fixes the values of $a_{3}, b_{3}$ and $c_{3}$. The parameters for the tendon profiles of the continuous beams are given in Table A1. 
Table A1. Parameters of tendon profiles. $h$ and $\max \{u(x)\}$ are the eccentricities at the lowest and highest point, respectively.

\begin{tabular}{|c|c|c|c|c|c|c|c|c|c|c|}
\hline$r$ & $\begin{array}{c}t \\
\mathrm{~m}\end{array}$ & $\begin{array}{l}h \\
\mathrm{~m}\end{array}$ & $\begin{array}{c}a_{l} \\
\mathrm{~m}^{-1}\end{array}$ & $b_{1}$ & $\begin{array}{c}a_{2} \\
\mathrm{~m}^{-1}\end{array}$ & $c_{2}$ & $\begin{array}{c}a_{3} \\
\mathrm{~m}^{-1}\end{array}$ & $b_{3}$ & $\begin{array}{l}c_{3} \\
\mathrm{~m}\end{array}$ & $\max \{u(x)\} / h$ \\
\hline \multirow[t]{3}{*}{6.393} & 6.500 & 0.50 & 0.00415 & -0.091 & 0.00375 & -0.50 & 0.0080 & 0.105 & 0.161 & 1.009 \\
\hline & & 1.00 & 0.00829 & -0.182 & 0.00750 & -1.00 & 0.0160 & 0.209 & 0.322 & 1.009 \\
\hline & & 2.00 & 0.01659 & -0.364 & 0.01500 & -2.00 & 0.0320 & 0.419 & 0.644 & 1.009 \\
\hline 4.911 & 5.000 & 2.00 & 0.01553 & -0.352 & 0.01350 & -2.00 & 0.0420 & 0.427 & 0.930 & 1.007 \\
\hline \multirow[t]{3}{*}{3.931} & 4.000 & 0.50 & 0.00370 & -0.086 & 0.00313 & -0.50 & 0.0130 & 0.107 & 0.272 & 0.983 \\
\hline & & 1.00 & 0.00740 & -0.172 & 0.00625 & -1.00 & 0.0261 & 0.214 & 0.545 & 0.983 \\
\hline & & 2.00 & 0.01481 & -0.344 & 0.01250 & -2.00 & 0.0522 & 0.428 & 1.090 & 0.983 \\
\hline
\end{tabular}

